\title{
PTU-008 SACRAL NERVE STIMULATION IN NEUROPATHIC CONSTIPATION
}

doi:10.1136/gut.2011.239301.136

U Khan, ${ }^{1 *} \mathrm{~J}$ Mason, ${ }^{2} \mathrm{P}$ Hungin, ${ }^{2} \mathrm{~J}$ Varma, ${ }^{1}$ Y Yiannakou' ${ }^{1}$ University Hospital of North Durham, Durham, UK; ${ }^{2}$ University of Durham, Durham, UK

Introduction The prevalence of constipation is between $43 \%$ and $58 \%$ in patients with either MS or spinal cord injury (SCI). Neuro-constipation is a very difficult to treat because of poor mobility, poor diet and side effects of long-term medication. Surgical options for intractable neuroconstipation involve prolonged operations with significant complications, and are unacceptable to many patients. In contrast sacral nerve stimulation (SNS) offers a potential minimally invasive and effective alternative.

Methods Patients with refractory neuropathic constipation were offered temporary SNS (TSNS).Bilateral TSNS was performed under sedation and electrodes placed in the sacral foramen that produced the best peripheral response. The electrodes were removed after a 3 week evaluation period (week 7, 8 and 9 of the study) during which patients completed diaries and questionnaires. Patients with more than $50 \%$ improvement of baseline symptoms and recurrence of pre-stimulation symptoms were offered bilateral permanent SNS (PSNS). Paired student $t$ test was used to calculate the changes in outcome measures before and during treatment.

Results 22 patients recruited in the trial over 2 years. There were 8 men and 14 women with an average age of 51.5 years (range 38-69 years.). 4 patients dropped out and 18 completed 


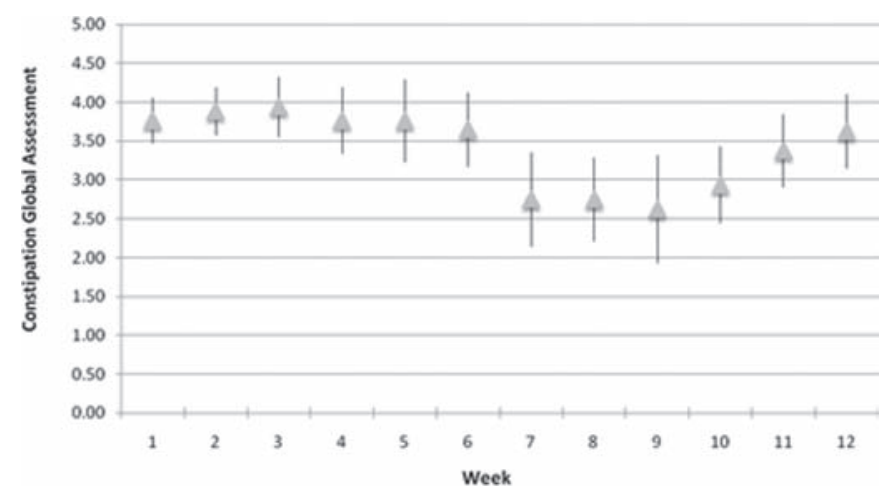

Figure 1 PTU-008

the trial. 12 patients $(67 \%$ ) received a permanent implant. The Global Assessment Score for constipation, PAC-SYM and PAC-QOL scores for these patients improved during treatment ( $p$ value $<0.05)$. There was also an improvement in toileting time ( $p 0.04$ ) and decrease in overall laxative use ( $p$ 0.03). 6 of the patients with a permanent implant had a tailed response to long-term stimulation and their symptoms have returned to baseline. Long-term response after PSNS was $33 \%(6 / 18)$.

Conclusion Patients with neuropathic constipation have a better response after TSNS than patients with idiopathic constipation. However TSNS has only a $50 \%$ positive predictive value for a long-term response. SNS offers a reasonable alternative to invasive surgical procedures in these patients but method of predicting long term response need to be improved.

Competing interests None. 